xxvii

\title{
Note on a special determinant
}

By A. C. Artken, University of Edinburgh.

Suppose a polynomial or convergent power series

$$
f(x)=a_{0}+a_{1} x+a_{2} x^{2}+a_{3} x^{3}+\ldots
$$

is raised to powers $j=0,1,2,3, \ldots$. The coefficients of $x^{k}$ in $[f(x)]^{j}, k=0,1,2, \ldots$, may be entered as elements in positions $(j, k)$ in an array or matrix $F$, thus:

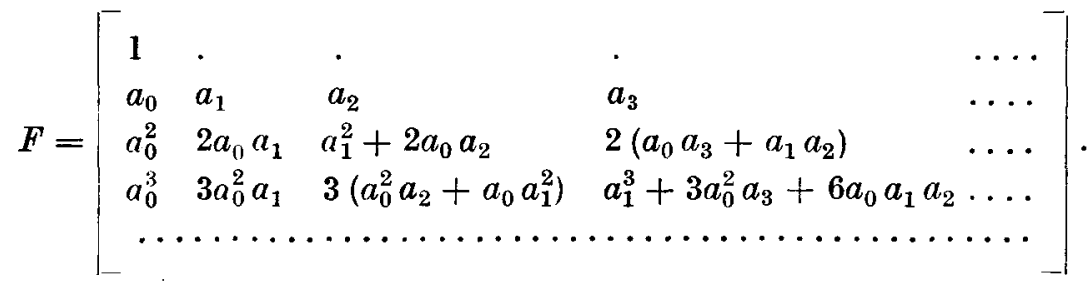

By construction all elements in column $(k)$ have weight (sum of suffixes) equal to $k$.

The array $F$ has interesting properties, which have been considered in detail by H. W. Turnbull, Proc. London Math. Soc. 37 (1934), 106-146. The reciprocal array $F^{-1}$ corresponds to the reversion of the series (1). One of the theorems proved (p. 121) is that the determinant $\left|F^{\prime}\right|_{n}$ obtained by taking the first $n$ rows and columns of $F$ has the value $a_{1}^{\frac{1}{2} n(n-1)}$.

The following simple proof of this may be put on record. Consider $\left[f(x)-a_{0}\right]^{j}$; it does not contain $a_{0}$. Translating this operation on $f(x)$, for $j=0,1,2, \ldots$, into an operation on $F$, we have at once

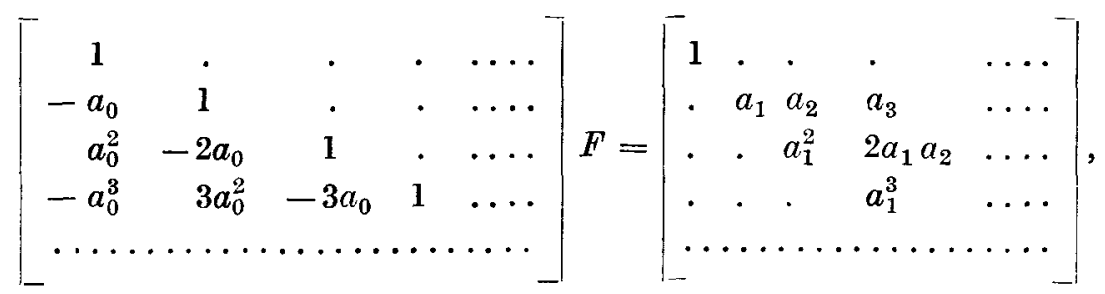

the right-hand array being $F$ with $a_{0}$ obliterated. Taking now determinants of both sides, we have

$$
|F|_{n}=a_{1}^{0+1+2+\ldots .+(n-1)}=a_{1}^{\frac{1}{n}(n-1)} .
$$


xxviii

We may prove in the same way that if $F_{m}$ is the array formed from the rows of $F$ beginning at $j=m$ instead of $j=0$, then the determinant formed from the first $n$ rows and columns of $F_{m}$ has the value

$$
\left|F_{m}\right|_{n}=a_{0}^{m n} a_{1}^{\frac{3}{n} n(n-1)} \text {. }
$$

To prove this we observe that $[f(x)]^{m}\left[f(x)-a_{0}\right]^{j}$, with $m$ fixed, $j=0,1,2, \ldots$, can possess no power of $a_{0}$ higher than $a_{0}^{m}$. Obliterating from $F_{m}$ such higher powers, we have

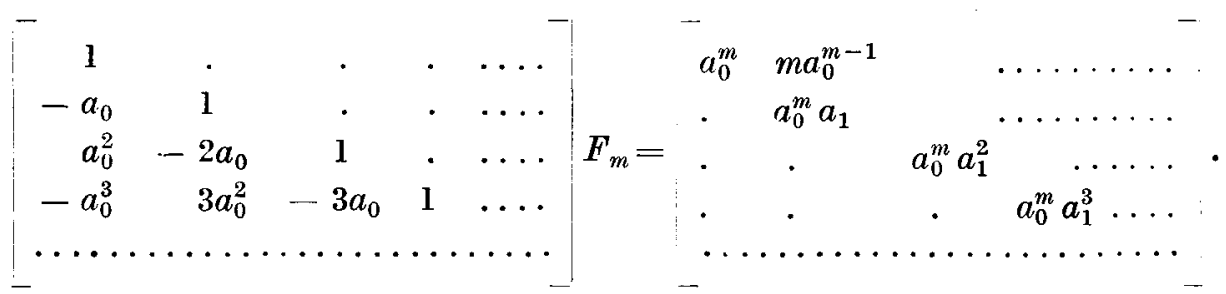

It follows as before that

$$
\left|F_{m}\right|_{n}=a_{0}^{m n} a_{1}^{\frac{1}{n} n(n-1)}
$$

The substitution of various special functions such as $e^{a x},(1+a x)^{p}$, and so on for $f(x)$ gives nothing very new, mostly variations on the old theme, that the difference-product of the numbers $0,1,2, \ldots, n$ is $n !(n-1) ! \ldots 3 ! 2 ! 1 !$ or $1^{n} 2^{n-1} 3^{n-2} \ldots n^{1}$, or the equally old theme, that the difference-product of $0,1, \frac{1}{2}, \frac{1}{3}, \ldots, \frac{1}{n}$ is $\left(1^{1} 2^{2} 3^{3} \ldots n^{n}\right)^{-1}$. 